\title{
Workless households for areas across the UK in 2010
}

\section{Coverage: UK}

Date: 08 September 2011

Geographical Area: Local Authority and County

Theme: Labour Market

\section{Animated YouTube Video}

A short video podcast explaining this story using audio commentary and graphical animations is available on the ONS YouTube channel at http://www.youtube.com/user/ onsstats? feature $=$ mhee\#p/u/0/mUQjbjM5enM

\section{Workless households across the UK by area}

Highest percentage of workless households across the UK in Liverpool

In 2010, there were three areas across the UK where more than three out of every 10 households had no-one in work, according to sub-regional data on working and workless households. These were:

- Liverpool (31.9 per cent);

- Nottingham (31.6 per cent) and

- Glasgow City (30.7 per cent).

Percentage of workless households, NUTS3 areas, January to December 2010 


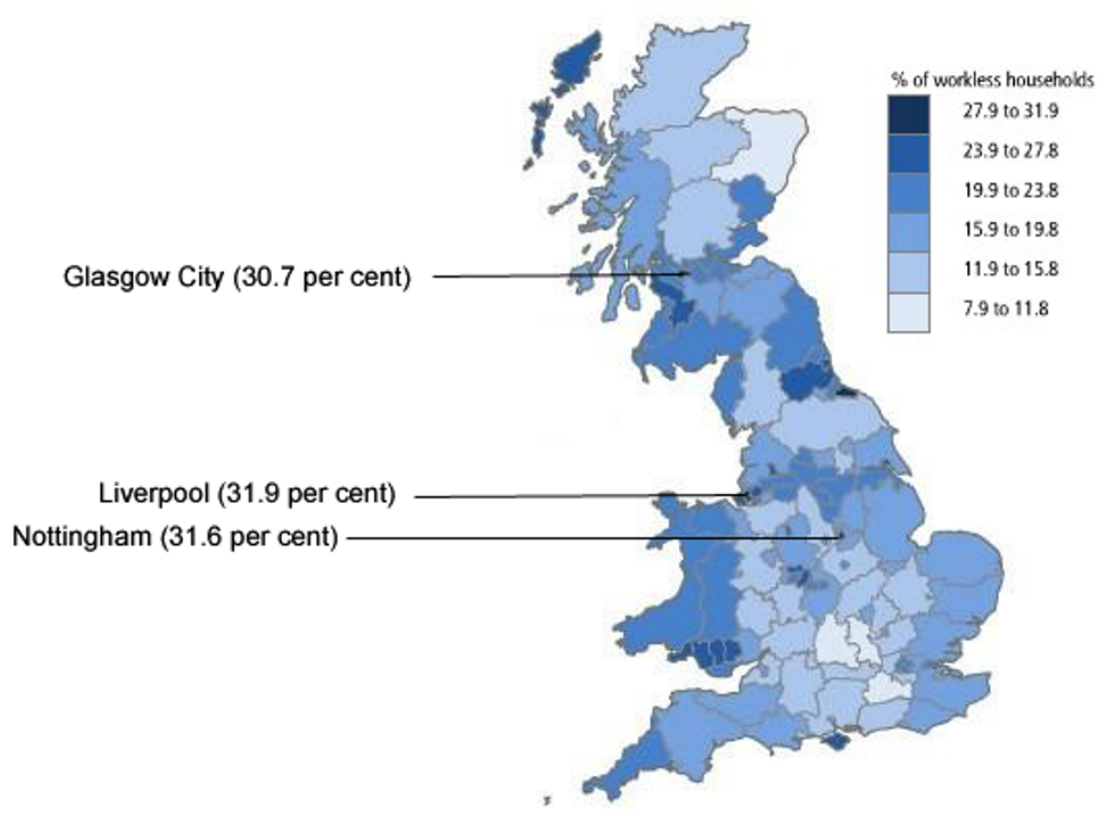

This was the second consecutive year that these three areas had the highest percentage of workless households, although for Liverpool and Glasgow City the percentage fell, from 32.1 per cent and 31.1 per cent respectively. In Nottingham the percentage of workless households increased from 31.3 per cent. Over the seven years since 2004 that data are available, Liverpool has had the highest percentage of workless households in five of the years, with it being in the top three in the other two years.

There were differences in the reasons why members of workless households in the top three said they were not working. Being sick or disabled (at 28 per cent) was the main reason for such people nationally to not be in work, and this was also the percentage in Liverpool, while 33 per cent gave this reason in Glasgow. However in Nottingham, partly because of its multiple universities, 43 per cent of people in workless households gave study as their reason, compared with 12 per cent nationally.

The areas with the lowest percentage of workless households in 2010 were Oxfordshire and Surrey, each at 11.2 per cent, followed by Aberdeen City, Aberdeenshire and North East Moray at 11.3 per cent. This equates to around one in every nine households being workless as opposed to almost one in every three for those areas with the highest percentage of workless households.

Over the same period for the UK as a whole, 18.9 per cent of households had no-one in work. The areas closest to the national average were Somerset (19.0 per cent) and Monmouthshire \& Newport (18.7 per cent).

Some people in workless households are not working because of early retirement. Nationally, 19 per cent of people aged 16 to 64 living in workless households gave this reason. The percentage was much higher in the South West at 27 per cent, with just 10 per cent in London. 
The 3.9 million workless households in the UK in 2010 contained 5.4 million people aged 16 to 64 . For the UK as a whole, the reasons for these people to not be in work were:

- Sick or disabled (28 per cent)

- Unemployed (19 per cent)

- Retired (19 per cent)

- Looking after the family or home (16 per cent)

- Study (12 per cent)

- Other (7 per cent)

A full breakdown of the reasons for each of the regions across the UK is in the supporting spreadsheet.

\section{Index of Tables}

Table A - Households by the combined economic activity status of household members by area

Table B - People by the combined economic activity status of household members by area

Table C - Children by the combined economic activity status of household members by area

Table D - Reasons for worklessness by region 


\section{North East}

Percentage of workless households in North East, January to December 2010

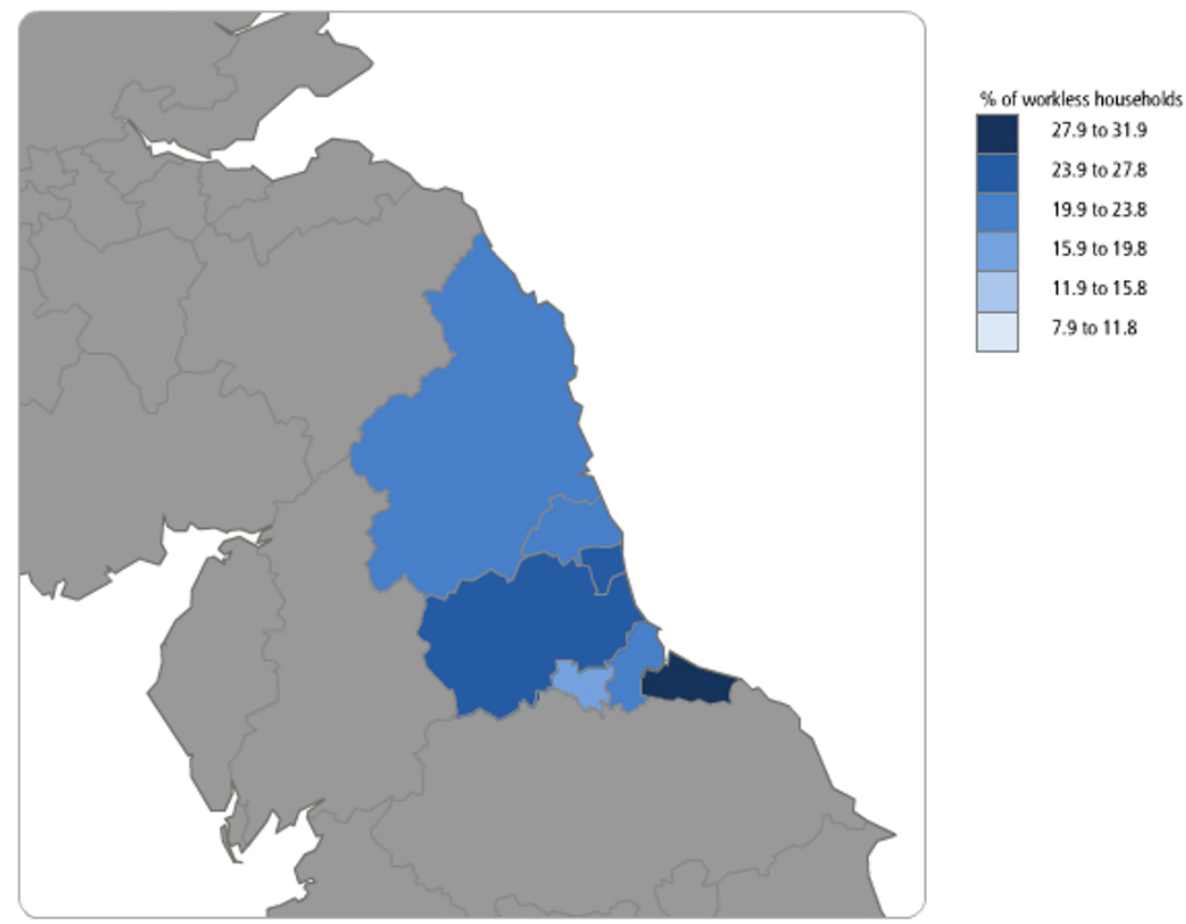

Workless households by NUTS3 area in North East, January to December 2010

\begin{tabular}{|c|c|c|c|c|}
\hline & & Area & Per cent & Thousands \\
\hline \multirow[t]{6}{*}{ Highest } & 1 & $\begin{array}{r}\text { South } \\
\text { Teesside }\end{array}$ & 29.2 & 27 \\
\hline & 2 & Sunderland & 26.0 & 24 \\
\hline & 3 & $\begin{array}{r}\text { Durham } \\
\text { CC }\end{array}$ & 25.0 & 45 \\
\hline & 4 & $\begin{array}{r}\text { Hartlepool } \\
\text { and } \\
\text { Stockton- } \\
\text { on-Tees }\end{array}$ & 22.9 & 21 \\
\hline & 5 & Tyneside & 22.7 & 64 \\
\hline & 6 & Northumberland & 21.7 & 22 \\
\hline Lowest & 7 & Darlington & 19.7 & 7 \\
\hline
\end{tabular}




\section{Download table}

\section{XLS XLS format}

\section{North West}

Percentage of workless households in North West, January to December 2010

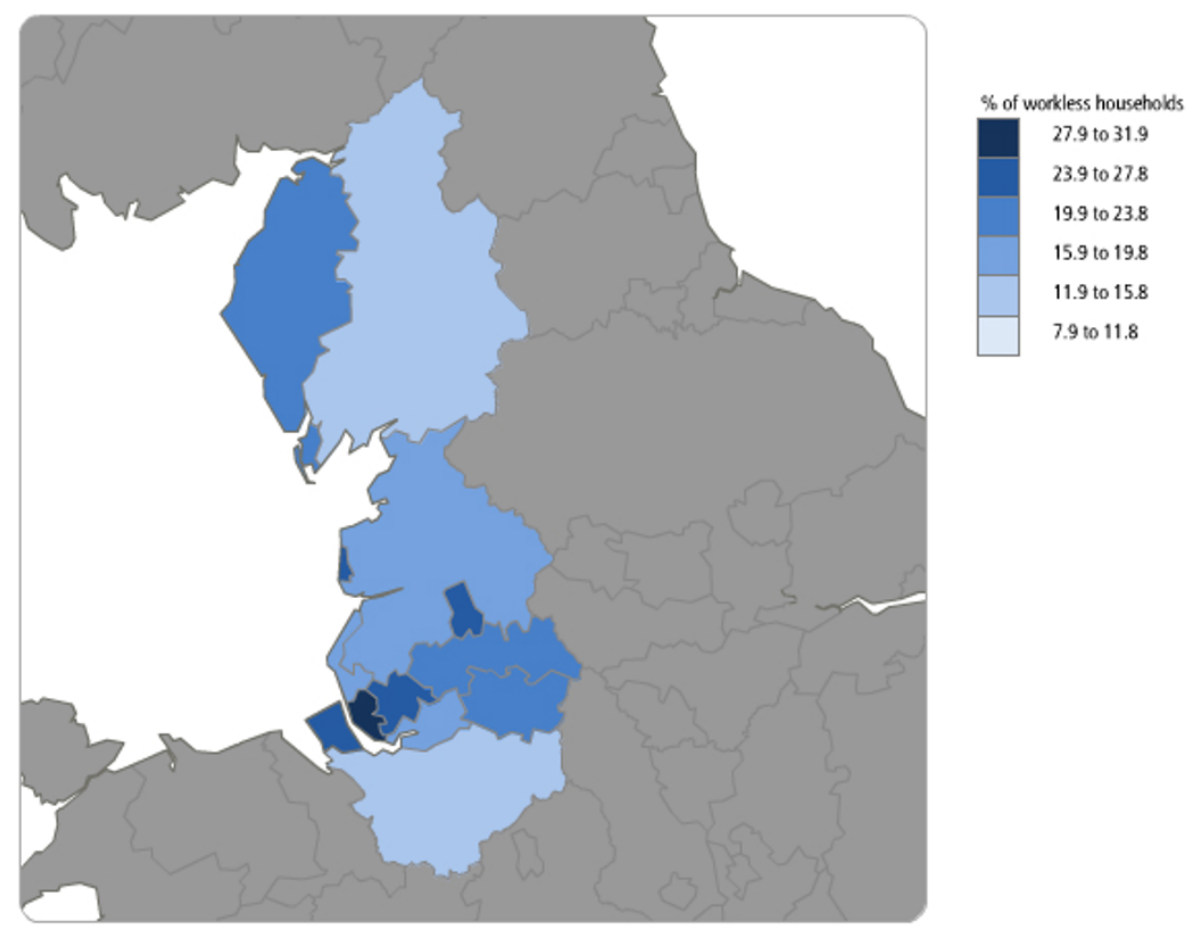


Workless households by NUTS3 area in North West, January to December 2010

\begin{tabular}{|c|c|c|c|c|}
\hline & & Area & Per cent & Thousands \\
\hline \multirow[t]{12}{*}{ Highest } & 1 & Liverpool & 31.9 & 51 \\
\hline & 2 & $\begin{array}{r}\text { East } \\
\text { Merseyside }\end{array}$ & 25.7 & 27 \\
\hline & 3 & Wirral & 25.3 & 26 \\
\hline & 4 & Blackpool & 25.0 & 12 \\
\hline & 5 & $\begin{array}{r}\text { Blackburn } \\
\text { with } \\
\text { Darwen }\end{array}$ & 24.9 & 11 \\
\hline & 6 & $\begin{array}{r}\text { Greater } \\
\text { Manchester } \\
\text { South }\end{array}$ & 22.2 & 108 \\
\hline & 7 & $\begin{array}{r}\text { Greater } \\
\text { Manchester } \\
\text { North }\end{array}$ & 20.9 & 82 \\
\hline & 8 & $\begin{array}{r}\text { West } \\
\text { Cumbria }\end{array}$ & 20.0 & 16 \\
\hline & 9 & Sefton & 19.3 & 17 \\
\hline & 10 & $\begin{array}{r}\text { Lancashire } \\
\mathrm{CC}\end{array}$ & 18.1 & 68 \\
\hline & 11 & $\begin{array}{r}\text { Halton } \\
\text { and } \\
\text { Warrington }\end{array}$ & 17.0 & 18 \\
\hline & 12 & $\begin{array}{r}\text { Cheshire } \\
\text { CC }\end{array}$ & 15.4 & 34 \\
\hline Lowest & 13 & $\begin{array}{r}\text { East } \\
\text { Cumbria }\end{array}$ & 13.6 & 12 \\
\hline
\end{tabular}

\section{Download table}

XLS XLS format 


\section{Yorkshire and the Humber}

Percentage of workless households in Yorkshire and the Humber, January to December 2010
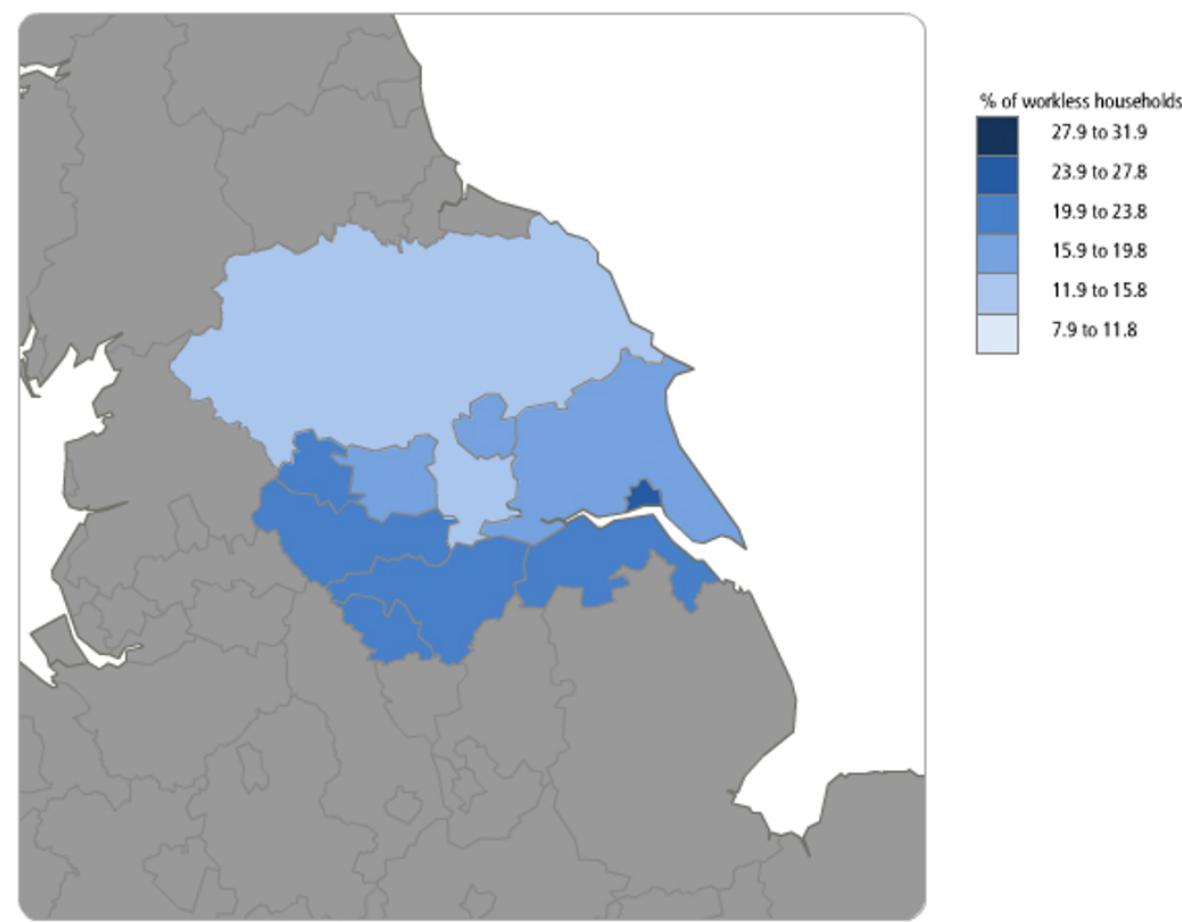

Workless households by NUTS3 area in Yorkshire and the Humber, January to December 2010

\begin{tabular}{|c|c|c|c|c|}
\hline & & Area & Per cent & Thousands \\
\hline \multirow[t]{5}{*}{ Highest } & 1 & $\begin{array}{r}\text { Kingston } \\
\text { upon } \\
\text { Hull, City } \\
\text { of }\end{array}$ & 26.8 & 25 \\
\hline & 2 & Bradford & 22.6 & 37 \\
\hline & 3 & $\begin{array}{r}\text { Barnsley, } \\
\text { Doncaster } \\
\text { and } \\
\text { Rotherham }\end{array}$ & 21.5 & 54 \\
\hline & 4 & Sheffield & 21.4 & 39 \\
\hline & 5 & $\begin{array}{r}\text { North and } \\
\text { North }\end{array}$ & 20.7 & 22 \\
\hline
\end{tabular}




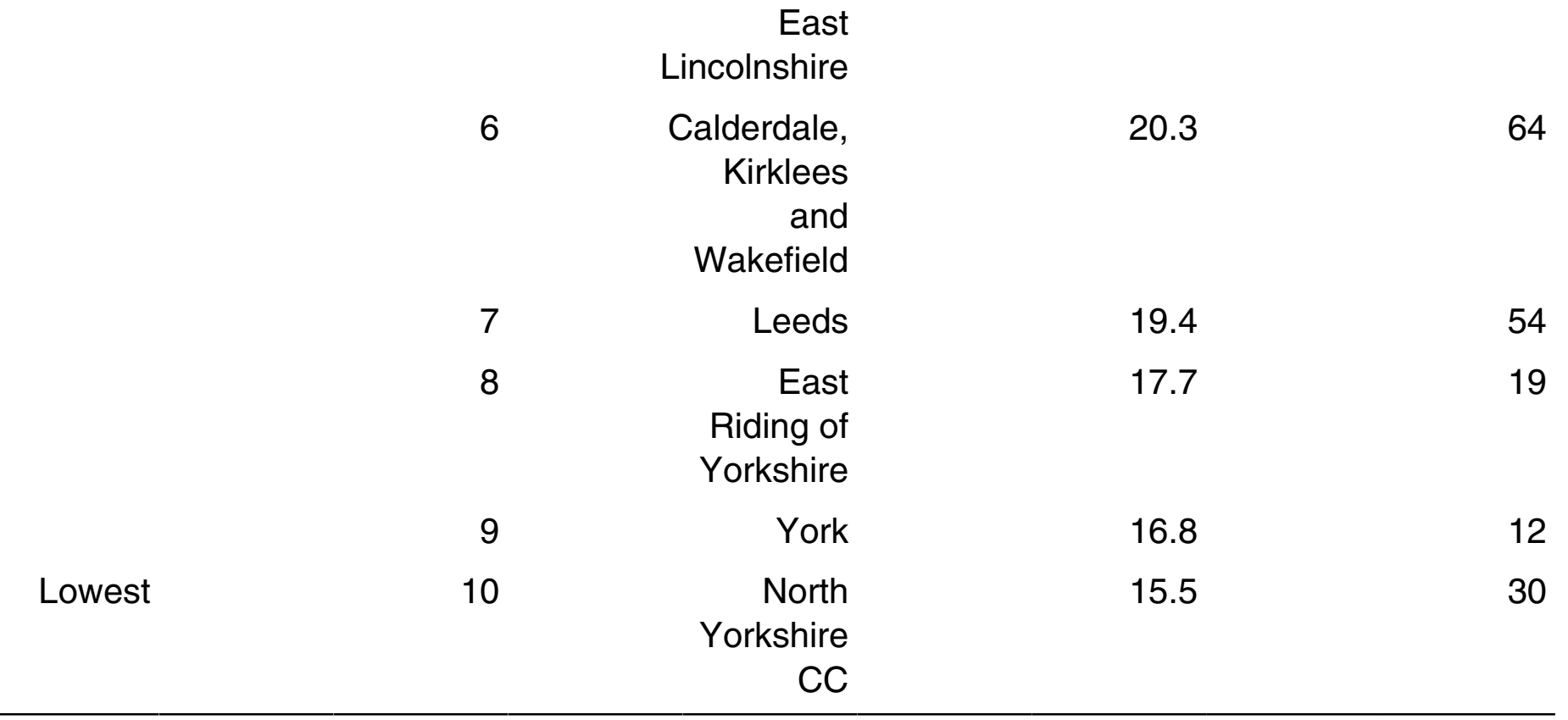

\section{Download table}

\section{XLS XLS format}

\section{East Midlands}

Percentage of workless households in East Midlands, January to December 2010

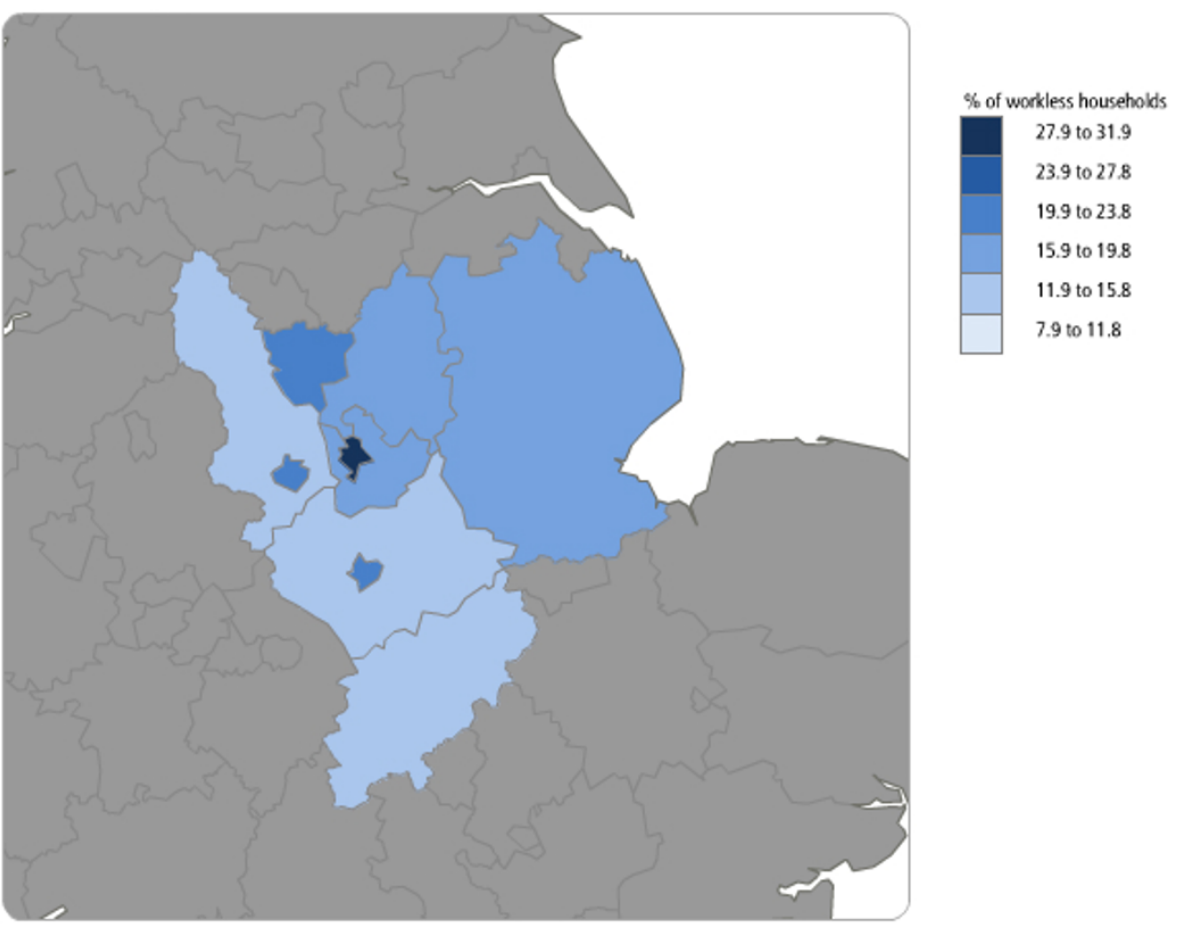


Workless households by NUTS3 area in East Midlands, January to December 2010

\begin{tabular}{|c|c|c|c|c|}
\hline & & Area & Per cent & Thousands \\
\hline \multirow[t]{9}{*}{ Highest } & 1 & Nottingham & 31.6 & 33 \\
\hline & 2 & Leicester & 22.2 & 22 \\
\hline & 3 & $\begin{array}{r}\text { East } \\
\text { Derbyshire }\end{array}$ & 21.0 & 19 \\
\hline & 4 & Derby & 19.9 & 16 \\
\hline & 5 & $\begin{array}{r}\text { North } \\
\text { Nottinghamshire }\end{array}$ & 17.9 & 26 \\
\hline & 6 & $\begin{array}{r}\text { South } \\
\text { Nottinghamshire }\end{array}$ & 17.0 & 18 \\
\hline & 7 & Lincolnshire & 16.6 & 37 \\
\hline & 8 & $\begin{array}{r}\text { Leicestershire } \\
\mathrm{CC} \text { and } \\
\text { Rutland }\end{array}$ & 14.9 & 32 \\
\hline & 9 & Northamptonshire & 14.1 & 33 \\
\hline Lowest & 10 & $\begin{array}{r}\text { South } \\
\text { and West } \\
\text { Derbyshire }\end{array}$ & 13.9 & 22 \\
\hline
\end{tabular}

\section{Download table}

XLS XLS format 


\section{West Midlands}

Percentage of workless households in West Midlands, January to December 2010

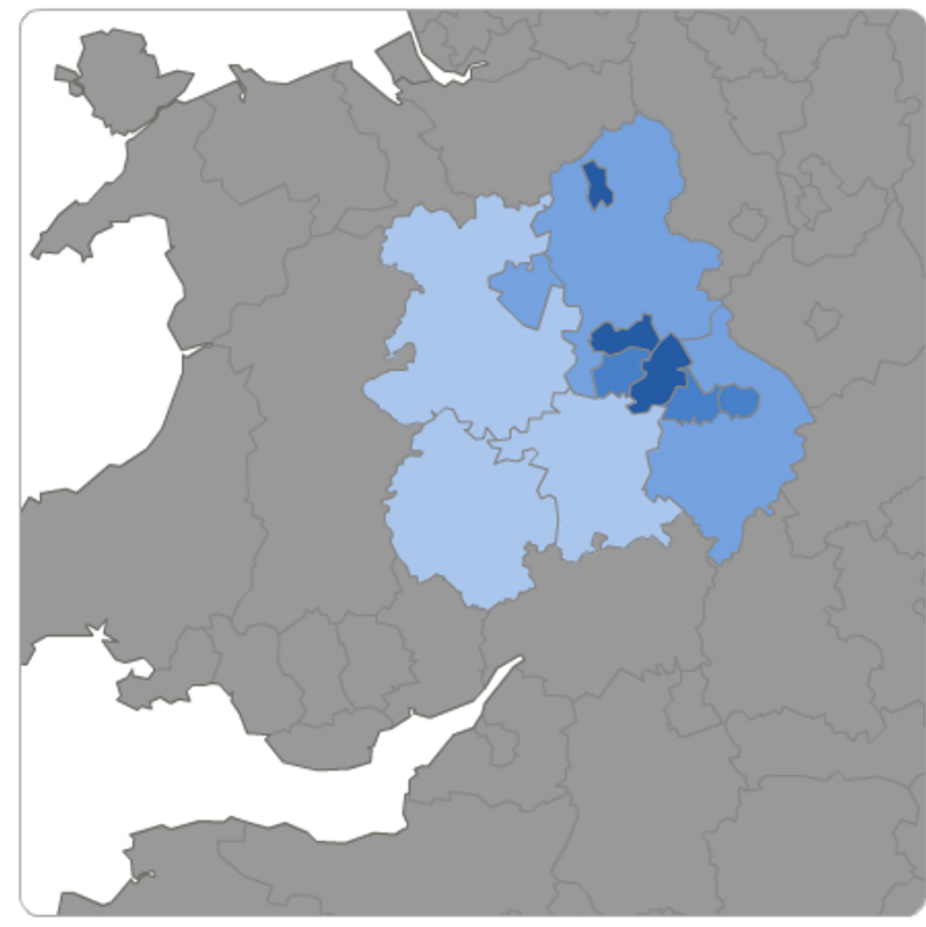

\begin{tabular}{|c|c|}
\hline$\%$ of workless households \\
27.9 to 31.9 \\
23.9 to 27.8 \\
19.9 to 23.8 \\
15.9 to 19.8 \\
11.9 to 15.8 \\
7.9 to 11.8
\end{tabular}


Workless households by NUTS3 area in West Midlands, January to December 2010

\begin{tabular}{|c|c|c|c|c|}
\hline & & Area & Per cent & Thousands \\
\hline \multirow[t]{11}{*}{ Highest } & 1 & Birmingham & 26.7 & 89 \\
\hline & 2 & $\begin{array}{r}\text { Walsall } \\
\text { and } \\
\text { Wolverhampton }\end{array}$ & 26.7 & 40 \\
\hline & 3 & $\begin{array}{r}\text { Stoke-on- } \\
\text { Trent }\end{array}$ & 25.1 & 21 \\
\hline & 4 & $\begin{array}{r}\text { Dudley } \\
\text { and } \\
\text { Sandwell }\end{array}$ & 23.5 & 45 \\
\hline & 5 & Coventry & 22.6 & 24 \\
\hline & 6 & Solihull & 20.9 & 13 \\
\hline & 7 & $\begin{array}{r}\text { Telford } \\
\text { and } \\
\text { Wrekin }\end{array}$ & 19.2 & 10 \\
\hline & 8 & Warwickshire & 16.3 & 28 \\
\hline & 9 & $\begin{array}{r}\text { Staffordshire } \\
\text { CC }\end{array}$ & 16.0 & 44 \\
\hline & 10 & $\begin{array}{r}\text { Herefordshire, } \\
\text { County of }\end{array}$ & 15.8 & 9 \\
\hline & 11 & Worcestershire & 15.7 & 28 \\
\hline Lowest & 12 & $\begin{array}{r}\text { Shropshire } \\
\text { CC }\end{array}$ & 13.3 & 13 \\
\hline
\end{tabular}

Download table

XLS XLS format 


\section{East of England}

Percentage of workless households in the East of England, January to December 2010
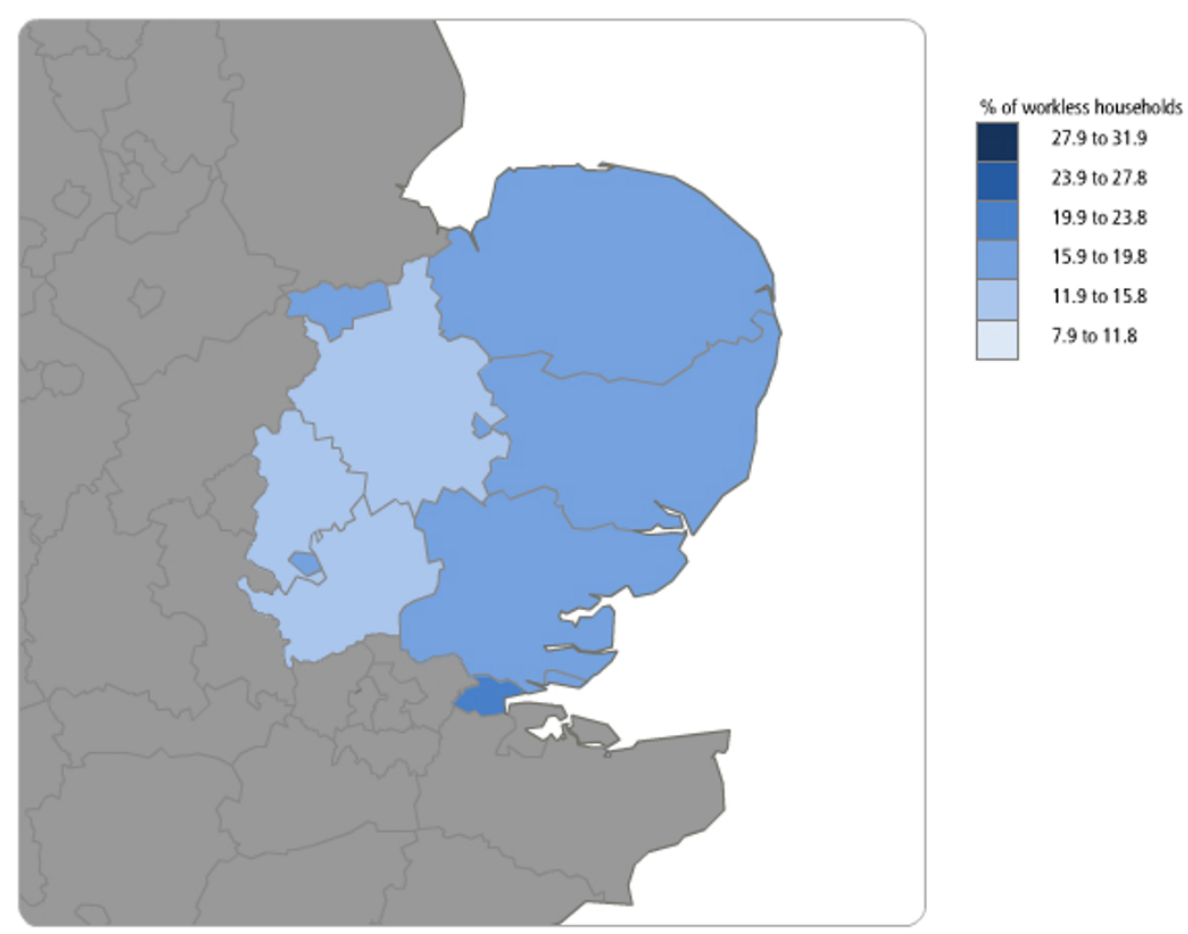

Workless households by NUTS3 area in East of England, January to December 2010

\begin{tabular}{|c|c|c|c|c|}
\hline & & Area & Per cent & Thousands \\
\hline \multirow[t]{9}{*}{ Highest } & 1 & Thurrock & 20.2 & 11 \\
\hline & 2 & Peterborough & 19.5 & 11 \\
\hline & 3 & Luton & 18.2 & 11 \\
\hline & 4 & $\begin{array}{l}\text { Southend- } \\
\text { on-Sea }\end{array}$ & 18.1 & 10 \\
\hline & 5 & Norfolk & 17.6 & 47 \\
\hline & 6 & Essex CC & 17.2 & 78 \\
\hline & 7 & Suffolk & 16.3 & 38 \\
\hline & 8 & $\begin{array}{r}\text { Cambridgeshire } \\
\mathrm{CC}\end{array}$ & 13.0 & 27 \\
\hline & 9 & Hertfordshire & 12.8 & 45 \\
\hline Lowest & 10 & $\begin{array}{r}\text { Bedfordshire } \\
\text { CC }\end{array}$ & 12.7 & 17 \\
\hline
\end{tabular}




\section{Download table}

\section{XLS XLS format}

\section{London}

Percentage of workless households in London, January to December 2010

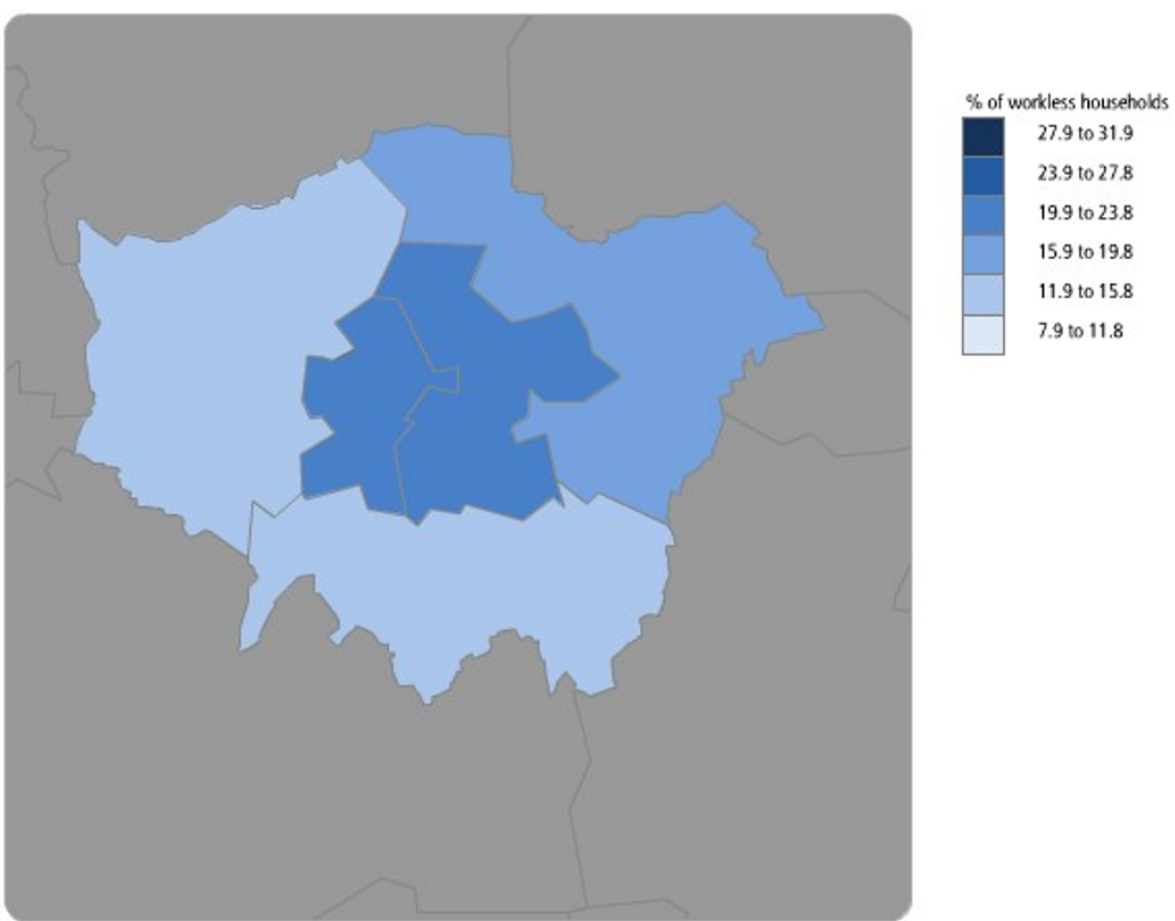

Workless households by NUTS3 area in London, January to December 2010

\begin{tabular}{|c|c|c|c|c|}
\hline & & Area & Per cent & Thousands \\
\hline \multirow[t]{4}{*}{ Highest } & 1 & $\begin{array}{r}\text { Inner } \\
\text { London - } \\
\text { West }\end{array}$ & 22.3 & 97 \\
\hline & 2 & $\begin{array}{r}\text { Inner } \\
\text { London - } \\
\text { East }\end{array}$ & 22.1 & 160 \\
\hline & 3 & $\begin{array}{r}\text { Outer } \\
\text { London } \\
- \text { East } \\
\text { and North } \\
\text { East }\end{array}$ & 19.4 & 105 \\
\hline & 4 & $\begin{array}{l}\text { Outer } \\
\text { London } \\
\text { - West }\end{array}$ & 14.9 & 87 \\
\hline
\end{tabular}




\begin{tabular}{crc} 
and North \\
West \\
Lowest & $\begin{array}{c}\text { Outer } \\
\text { London - } \\
\text { South }\end{array}$ & 14.9 \\
& & \\
\hline
\end{tabular}

\section{Download table}

\section{XLS XLS format}

\section{South East}

Percentage of workless households in South East, January to December 2010

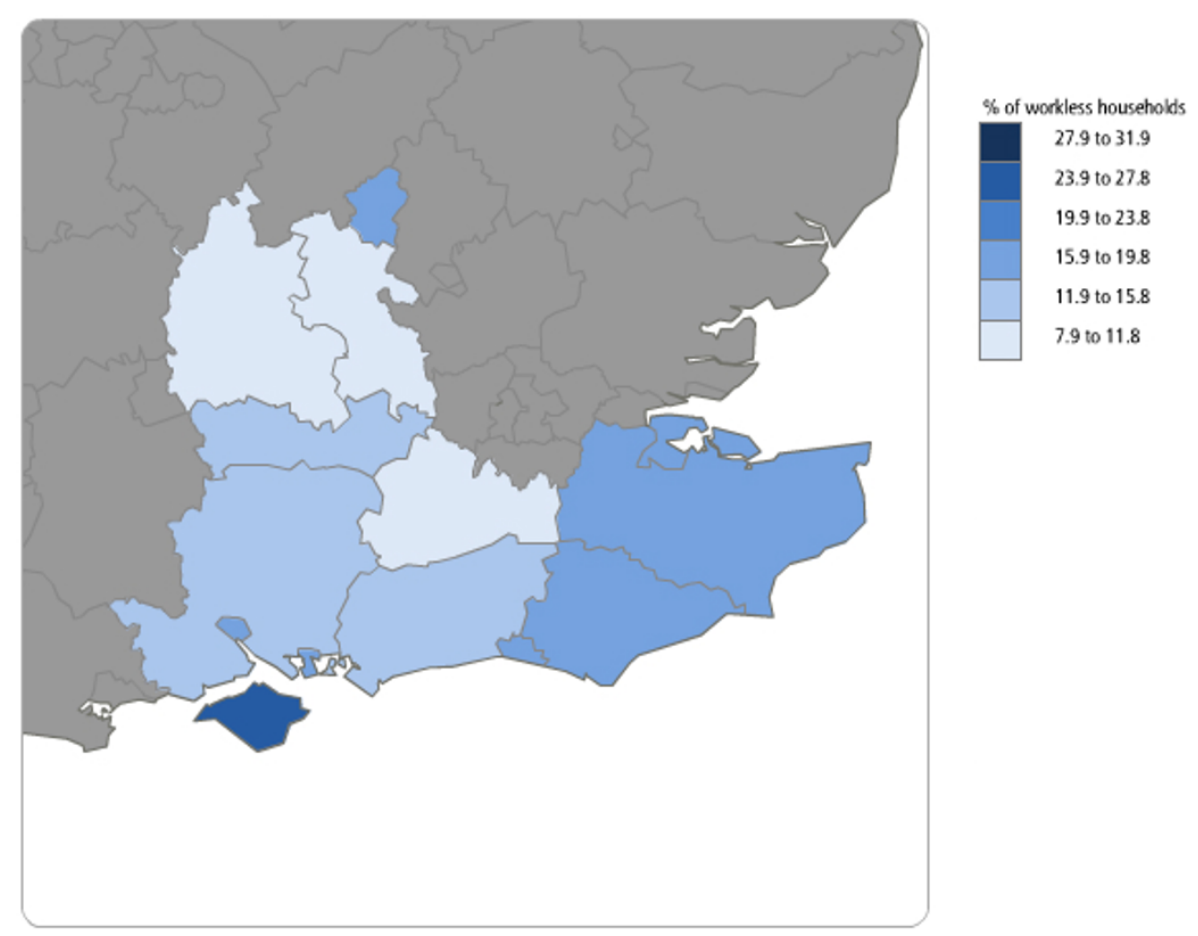


Workless households by NUTS3 area in South East, January to December 2010

\begin{tabular}{|c|c|c|c|c|}
\hline & & Area & Per cent & Thousands \\
\hline \multirow[t]{13}{*}{ Highest } & 1 & $\begin{array}{l}\text { Isle of } \\
\text { Wight }\end{array}$ & 26.6 & 11 \\
\hline & 2 & Southampton & 19.8 & 17 \\
\hline & 3 & Medway & 19.2 & 15 \\
\hline & 4 & $\begin{array}{r}\text { East } \\
\text { Sussex } \\
\text { CC }\end{array}$ & 18.0 & 29 \\
\hline & 5 & Kent CC & 17.9 & 79 \\
\hline & 6 & $\begin{array}{r}\text { Milton } \\
\text { Keynes }\end{array}$ & 17.6 & 14 \\
\hline & 7 & $\begin{array}{l}\text { Brighton } \\
\text { and Hove }\end{array}$ & 17.6 & 16 \\
\hline & 8 & Portsmouth & 17.1 & 12 \\
\hline & 9 & $\begin{array}{r}\text { West } \\
\text { Sussex }\end{array}$ & 13.7 & 35 \\
\hline & 10 & $\begin{array}{r}\text { Hampshire } \\
\text { CC }\end{array}$ & 12.5 & 52 \\
\hline & 11 & Berkshire & 12.1 & 34 \\
\hline & 12 & $\begin{array}{r}\text { Buckinghamshire } \\
\text { CC }\end{array}$ & 11.8 & 19 \\
\hline & 13 & Surrey & 11.2 & 41 \\
\hline Lowest & 14 & Oxfordshire & 11.2 & 23 \\
\hline
\end{tabular}

\section{Download table}

\section{XLS XLS format}




\section{South West}

Percentage of workless households in South West, January to December 2010

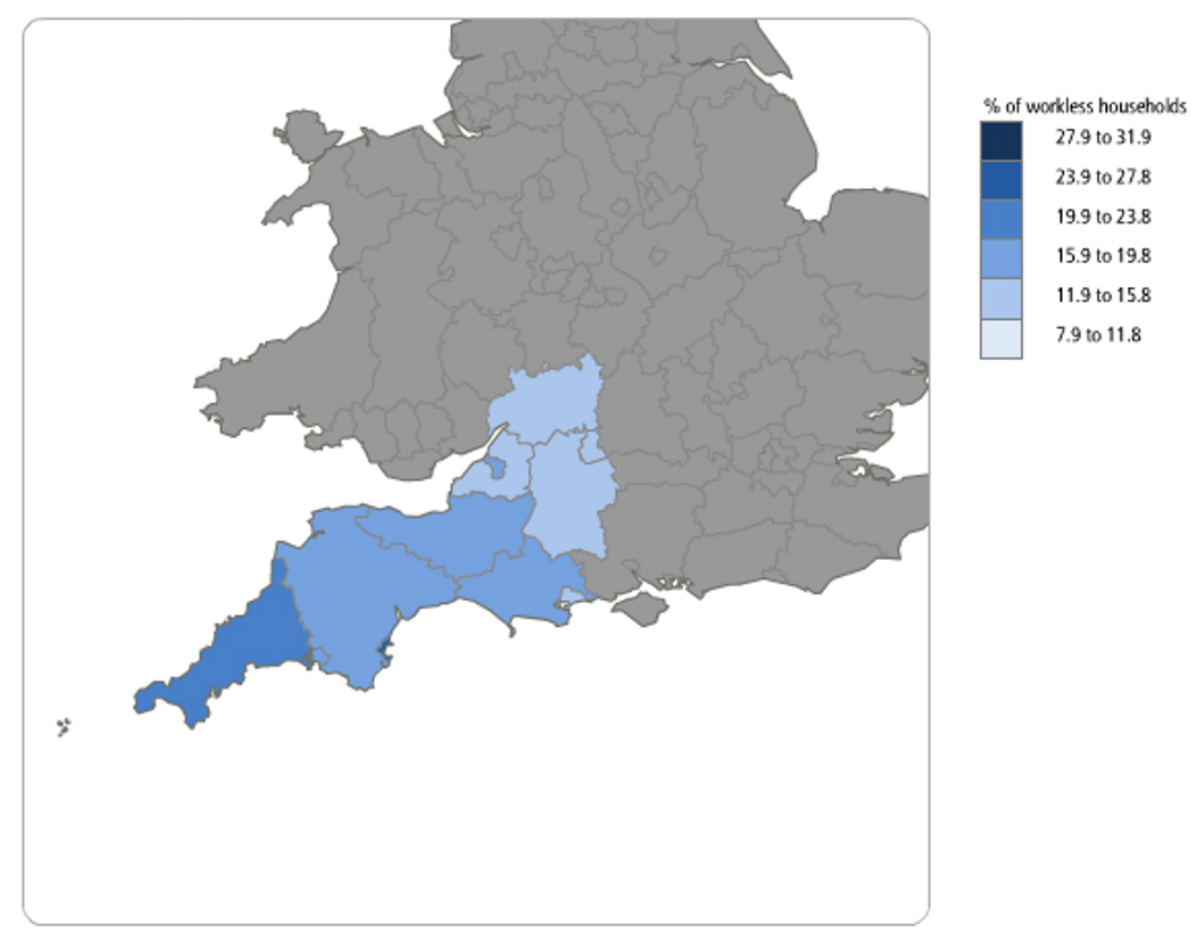


Workless households by NUTS3 area in South West, January to December 2010

\begin{tabular}{|c|c|c|c|c|}
\hline & & Area & Per cent & Thousands \\
\hline \multirow[t]{11}{*}{ Highest } & 1 & Torbay & 25.2 & 11 \\
\hline & 2 & $\begin{array}{r}\text { Cornwall } \\
\text { and Isles } \\
\text { of Scilly }\end{array}$ & 22.1 & 36 \\
\hline & 3 & Plymouth & 19.8 & 17 \\
\hline & 4 & Somerset & 19.0 & 32 \\
\hline & 5 & $\begin{array}{r}\text { Dorset } \\
\text { CC }\end{array}$ & 17.3 & 20 \\
\hline & 6 & $\begin{array}{l}\text { Bristol, } \\
\text { City of }\end{array}$ & 16.8 & 26 \\
\hline & 7 & $\begin{array}{r}\text { Devon } \\
\text { CC }\end{array}$ & 16.7 & 38 \\
\hline & 8 & $\begin{array}{r}\text { Bournemouth } \\
\text { and } \\
\text { Poole }\end{array}$ & 15.3 & 16 \\
\hline & 9 & $\begin{array}{r}\text { North and } \\
\text { North } \\
\text { East } \\
\text { Somerset, } \\
\text { South } \\
\text { Gloucestershire }\end{array}$ & 14.8 & 30 \\
\hline & 10 & Gloucestershire & 13.4 & 25 \\
\hline & 11 & Swindon & 12.3 & 8 \\
\hline Lowest & 12 & $\begin{array}{r}\text { Wiltshire } \\
\text { CC }\end{array}$ & 12.3 & 18 \\
\hline
\end{tabular}

\section{Download table}

XLS XLS format 


\section{Wales}

Percentage of workless households in Wales, January to December 2010

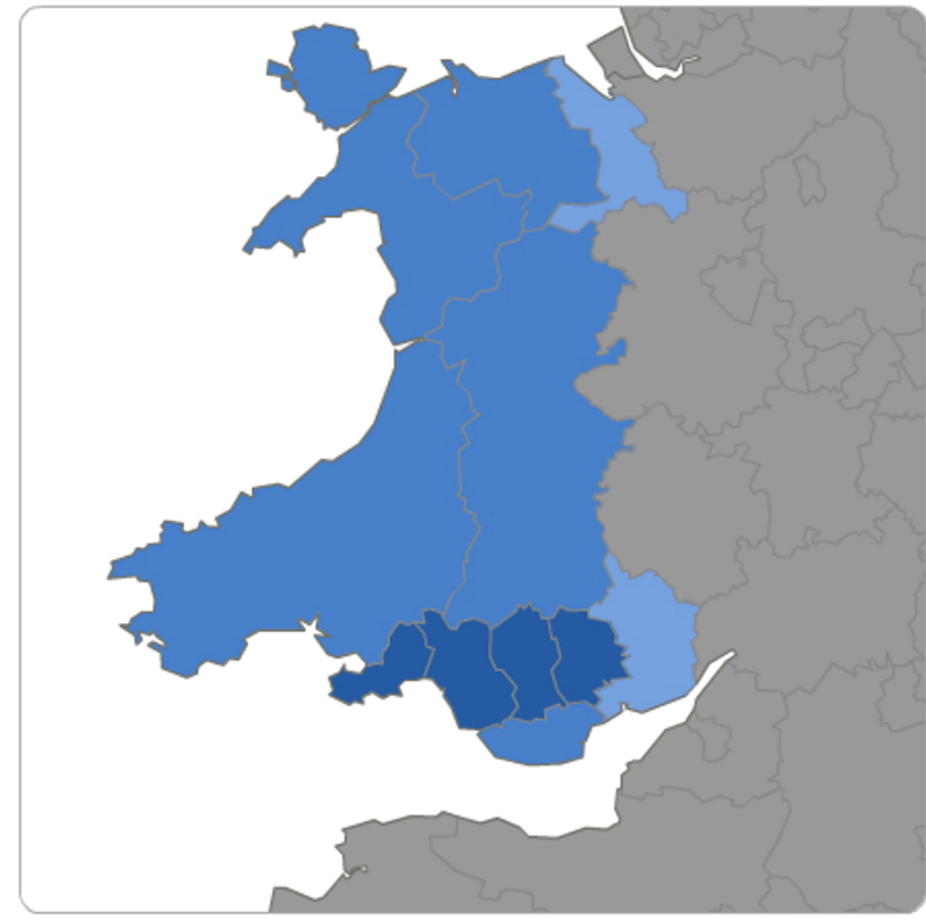

$$
\begin{array}{|}
\text { \% of workless households } \\
27.9 \text { to } 31.9 \\
23.9 \text { to } 27.8 \\
19.9 \text { to } 23.8 \\
15.9 \text { to } 19.8 \\
11.9 \text { to } 15.8 \\
7.9 \text { to } 11.8
\end{array}
$$

Workless households by NUTS3 area in Wales, January to December 2010

\begin{tabular}{lrrrr}
\hline \multirow{2}{*}{ Highest } & Area & & Per cent & Thousands \\
\cline { 3 - 3 } & 1 & Swansea & 26.6 & 20 \\
2 & $\begin{array}{r}\text { Central } \\
\text { Valleys }\end{array}$ & 26.5 & 26 \\
& 3 & $\begin{array}{r}\text { Bridgend } \\
\text { and } \\
\text { Neath }\end{array}$ & 26.3 & 24 \\
& $\begin{array}{r}\text { Neath } \\
\end{array}$ & &
\end{tabular}


Port

Talbot

4

Gwent

25.1

27

Valleys

5

Conwy

22.7

14

and

Denbighshire

6

South

21.7

26

West

Wales

7

Isle of

20.9

4

Anglesey

8

Cardiff

20.7

and

Vale of

Glamorgan

9

Powys

20.6

9

10

Gwynedd

20.5

7

11 Monmouthshire

18.7

14

and

Newport

Lowest

12

Flintshire

17.4

and

Wrexham

\section{Download table}

\section{XLS XLS format}




\section{Scotland}

Percentage of workless households in Scotland, January to December 2010

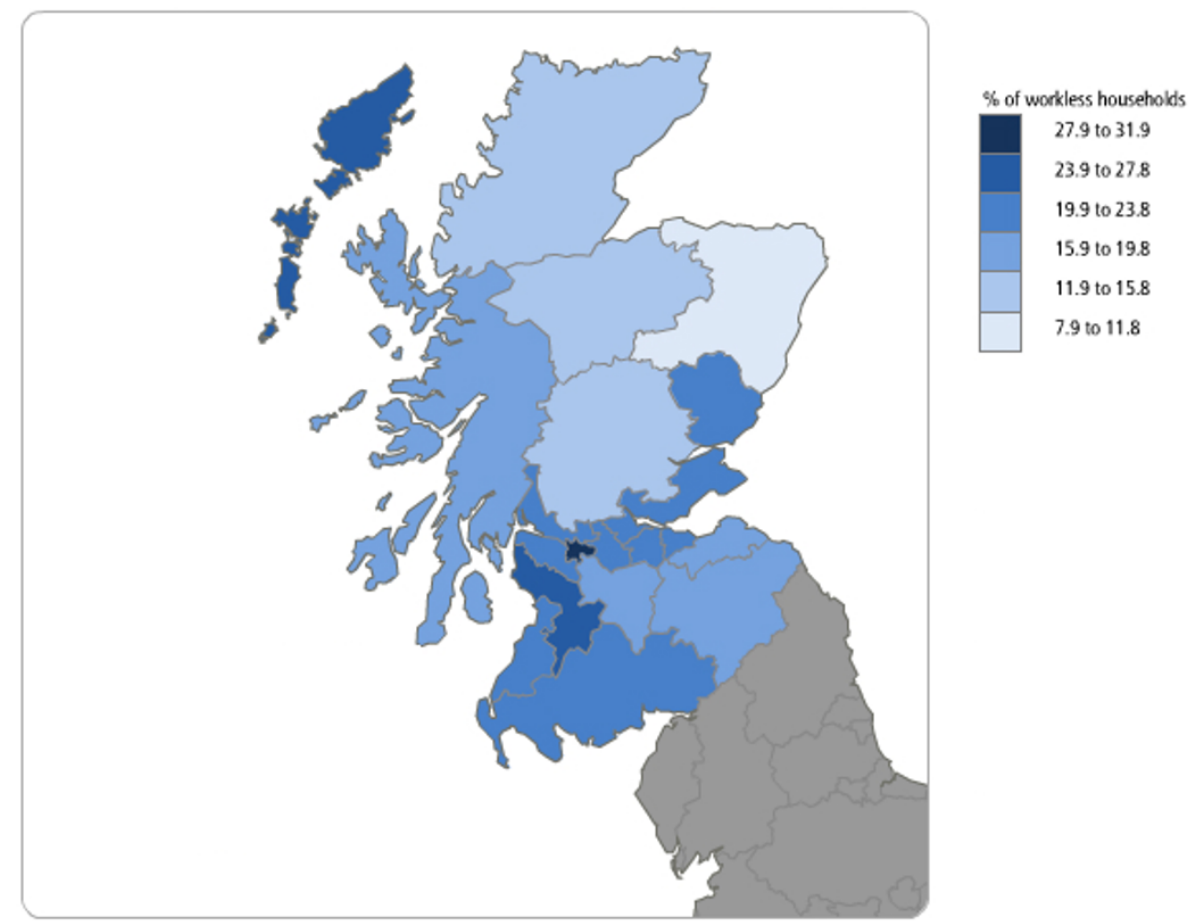

Workless households by NUTS3 area (new) in Scotland, January to December 2010

\begin{tabular}{|c|c|c|c|c|}
\hline & & Area & Per cent & Thousands \\
\hline \multirow[t]{3}{*}{ Highest } & 1 & $\begin{array}{r}\text { Glasgow } \\
\text { City }\end{array}$ & 30.7 & 72 \\
\hline & 2 & $\begin{array}{r}\text { East } \\
\text { Ayrshire } \\
\text { and North } \\
\text { Ayrshire } \\
\text { mainland }\end{array}$ & 27.3 & 24 \\
\hline & 3 & $\begin{array}{r}\text { Eilean } \\
\text { Siar }\end{array}$ & 25.3 & 2 \\
\hline
\end{tabular}


(Western

Isles)

4

North

23.6

26

Lanarkshire

5

South

22.6

9

Ayrshire

6

Inverclyde,

22.6

26

East

Renfrewshire

and

Renfrewshire

7

West

21.9

Lothian

8

Edinburgh,

21.4

40

9

City of

21.2

and

Dundee

City

Falkirk

20.5

11

11 Clackmannanshire

20.1

29

and Fife

12

East

20.0

14

Dunbartonshire,

West

Dunbartonshire

and

Helensburgh

\&

Lomond

13

Dumfries

19.9

10

Galloway

14

Lochaber,

Skye \&

Lochalsh,

Arran \&

Cumbrae

19.6 


\begin{tabular}{|c|c|c|c|}
\hline & $\begin{array}{l}\text { and Argyll } \\
\text { \& Bute }\end{array}$ & & \\
\hline 15 & $\begin{array}{l}\text { Scottish } \\
\text { Borders }\end{array}$ & 19.3 & 8 \\
\hline 16 & $\begin{array}{r}\text { East } \\
\text { Lothian } \\
\text { and } \\
\text { Midlothian }\end{array}$ & 18.4 & 11 \\
\hline 17 & $\begin{array}{r}\text { South } \\
\text { Lanarkshire }\end{array}$ & 18.4 & 21 \\
\hline 18 & $\begin{array}{r}\text { Perth \& } \\
\text { Kinross } \\
\text { and } \\
\text { Stirling }\end{array}$ & 15.6 & 12 \\
\hline 19 & $\begin{array}{r}\text { Caithness } \\
\& \\
\text { Sutherland } \\
\text { and } \\
\text { Ross \& } \\
\text { Cromarty }\end{array}$ & 14.2 & 4 \\
\hline 20 & $\begin{array}{r}\text { Inverness } \\
\& \text { Nairn } \\
\text { and } \\
\text { Moray, } \\
\text { Badenoch } \\
\& \\
\text { Strathspey }\end{array}$ & 11.9 & 7 \\
\hline 21 & $\begin{array}{r}\text { Aberdeen } \\
\text { City and } \\
\text { Aberdeenshire }\end{array}$ & 11.5 & 19 \\
\hline
\end{tabular}

\section{Table notes:}

1. This table gives estimates based on most recent NUTS3 area classification for Scotland. The following NUTS3 areas have changed from the old NUTS3 classification in boundary and in name: Aberdeen City and Aberdeenshire, Caithness \& Sutherland and Ross \& Cromarty, Inverness \& Nairn and Moray, Badenoch \& Strathspey, Lochaber, Skye \& Lochalsh, Arran \& Cumbrae and Argyll \& Bute.

\section{Download table}

\section{XLS XLS format}


Workless households by NUTS3 area (old) in Scotland, January to December 2010

\begin{tabular}{|c|c|c|c|c|}
\hline & & Area & Per cent & Thousands \\
\hline \multirow[t]{12}{*}{ Highest } & 1 & $\begin{array}{r}\text { Glasgow } \\
\text { City }\end{array}$ & 30.7 & 72 \\
\hline & 2 & $\begin{array}{r}\text { East } \\
\text { Ayrshire } \\
\text { and North } \\
\text { Ayrshire } \\
\text { mainland }\end{array}$ & 27.3 & 24 \\
\hline & 3 & $\begin{array}{r}\text { Eilean } \\
\text { Siar } \\
\text { (Western } \\
\text { Isles) }\end{array}$ & 25.3 & 2 \\
\hline & 4 & $\begin{array}{r}\text { North } \\
\text { Lanarkshire }\end{array}$ & 23.6 & 26 \\
\hline & 5 & $\begin{array}{r}\text { Inverclyde, } \\
\text { East } \\
\text { Renfrewshire } \\
\text { and } \\
\text { Renfrewshire }\end{array}$ & 22.6 & 26 \\
\hline & 6 & $\begin{array}{r}\text { South } \\
\text { Ayrshire }\end{array}$ & 22.6 & 9 \\
\hline & 7 & $\begin{array}{r}\text { West } \\
\text { Lothian }\end{array}$ & 21.9 & 13 \\
\hline & 8 & $\begin{array}{l}\text { Edinburgh, } \\
\text { City of }\end{array}$ & 21.4 & 40 \\
\hline & 9 & $\begin{array}{r}\text { Angus } \\
\text { and } \\
\text { Dundee } \\
\text { City }\end{array}$ & 21.2 & 19 \\
\hline & 10 & Falkirk & 20.5 & 11 \\
\hline & 11 & $\begin{array}{r}\text { Clackmannanshire } \\
\text { and Fife }\end{array}$ & 20.1 & 29 \\
\hline & 12 & $\begin{array}{r}\text { East and } \\
\text { West } \\
\text { Dunbartonshire, } \\
\text { Helensburgh }\end{array}$ & 20.0 & 14 \\
\hline
\end{tabular}




\begin{tabular}{|c|c|c|c|}
\hline 13 & $\begin{array}{r}\text { Dumfries } \\
\& \\
\text { Galloway }\end{array}$ & 19.9 & 10 \\
\hline 14 & $\begin{array}{l}\text { Lochaber, } \\
\text { Skye and } \\
\text { Lochalsh } \\
\text { and Argyll } \\
\text { and the } \\
\text { Islands }\end{array}$ & 19.8 & 5 \\
\hline 15 & $\begin{array}{l}\text { Scottish } \\
\text { Borders }\end{array}$ & 19.3 & 8 \\
\hline 16 & $\begin{array}{r}\text { East } \\
\text { Lothian } \\
\text { and } \\
\text { Midlothian }\end{array}$ & 18.4 & 11 \\
\hline 17 & $\begin{array}{r}\text { South } \\
\text { Lanarkshire }\end{array}$ & 18.4 & 21 \\
\hline 18 & $\begin{array}{r}\text { Perth \& } \\
\text { Kinross } \\
\text { and } \\
\text { Stirling }\end{array}$ & 15.6 & 12 \\
\hline 19 & $\begin{array}{r}\text { Caithness } \\
\text { and } \\
\text { Sutherland } \\
\text { and } \\
\text { Ross and } \\
\text { Cromarty }\end{array}$ & 14.3 & 4 \\
\hline 20 & $\begin{array}{r}\text { Inverness } \\
\text { and Nairn } \\
\text { and } \\
\text { Moray, } \\
\text { Badenoch } \\
\text { and } \\
\text { Strathspey }\end{array}$ & 12.9 & 5 \\
\hline 21 & $\begin{array}{r}\text { Aberdeen } \\
\text { City, } \\
\text { Aberdeenshire } \\
\text { and North }\end{array}$ & 11.3 & 21 \\
\hline
\end{tabular}


East

Moray

\section{Table notes:}

1. This table gives estimates based on old NUTS3 area classification for Scotland.

\section{Download table}

\section{XLS XLS format}

\section{Appendices}

\section{Background information}

Email: Labour.market@ons.gov.uk

\section{Concepts and definitions}

1. Estimates within this analysis only cover households that contain at least one person aged 16 to 64.

2. A household is defined as a single person, or a group of people living at the same address who have the address as their only or main residence and either share one main meal a day or share living accommodation (or both).

3. A working household is a household that contains at least one person aged 16 to 64 , where all individuals aged 16 and over are in employment.

4. A mixed household is a household that contains at least one person aged 16 to 64 , where at least one person aged 16 and over is in employment and at least one other is either unemployed or inactive.

5. A workless household is a household that contains at least one person aged 16 to 64 , where no-one aged 16 or over is in employment. 